\title{
FDG-PET and CT features of non-small cell lung cancer based on tumor type
}

\author{
SUZANNE L. AQUINO, ELKAN F. HALPERN, LANDON B. KUESTER and ALAN J. FISCHMAN
}

Department of Radiology, Massachusetts General Hospital, 55 Fruit Street, FND 202, Boston, MA 02114, USA

Received September 29, 2006; Accepted November 9, 2006

\begin{abstract}
We determined if specific tumor types of nonsmall cell lung cancer can be identified by variance in FDGPET standard uptake value (SUV) in combination with characteristics on CT. Staging FDG-PET and CT scans of 81 patients (34 men and 47 women, average age $67 \pm 11$ years) with 82 lung cancers were analyzed. Mean tumor SUV was calculated at the location of maximum FDG uptake. Tumor size, margins, and location were analyzed on CT. Statistical analysis compared SUV between tumor subtypes, assessed relationship between tumor subtype and features on CT and determined if combination of CT and SUV patterns predicted tumor type. In total 35 adenocarcinomas (AC); 15 bronchioloalveolar cell carcinomas (BAC), 23 squamous cell carcinomas and 9 large cell carcinomas were evaluated. Significant differences were found between SUV of all AC and squamous cell $(\mathrm{p}<0.0001)$; between all $\mathrm{AC}$ and large cell $(\mathrm{p}=0.03)$; between non-BAC AC and squamous cell types $(\mathrm{p}=0.0005)$; BAC and non-BAC AC $(\mathrm{p}=0.04), \mathrm{BAC}$ and squamous cell $(p<0.0001)$; BAC and large cell $(p=0.004)$. Ground glass was the most significant CT feature in distinguishing tumor types, which was seen in BAC $(\mathrm{p}<0.0003)$. In conclusion, SUVs for non-small cell lung cancer were most significantly different between BAC and all other NCLC cell subtypes. The presence of ground glass in a nodule on CT is a significant feature for BAC and should raise the suspicion for this tumor type despite low FDG uptake.
\end{abstract}

\section{Introduction}

PET with ${ }^{18}$ F-FDG has been shown to be useful in the detection of malignancy in pulmonary nodules (1-5). FDG, a glucose analog, is taken up by metabolically active cells and phosphorylated by the enzyme hexokinase. Many tumor cells are deficient in the enzyme, glucose-6-phosphatase, which converts FDG-6-phosphate back to FDG. Therefore, tumor cells trap

Correspondence to: Dr Alan J. Fischman, Massachusetts General Hospital, Department of Radiology, 55 Fruit Street, FND 202, Boston, MA 02114, USA

E-mail: fischman@pet.mgh.harvard.edu

Key words: lung neoplasm, carcinoma non-small cell lung, carcinoma, bronchioloalveolar, ${ }^{18} \mathrm{~F}-\mathrm{FDG}$, computerized emission tomography and accumulate this metabolite, FDG-6-phosphate, relative to other cells in the body and thus show greater FDG accumulation.

Prior studies have described a relatively greater FDG accumulation in lung tumors based on degree of differentiation (6). Higashi et al found that adenocarcinomas of poor differentiation showed greater uptake of FDG compared to well-differentiated adenocarcinomas such as BAC. Other studies have shown variable uptake including an absence of FDG uptake in primary bronchoalveolar cell carcinomas and carcinoid tumors of the lung (7-10). By using the accepted SUV value cutoff of 2.5 , those tumors of lower values will be interpreted as 'non-malignant'.

Even in the absence of increased FDG uptake, some features on CT should still raise a suspicion for tumor. For instance a part-solid nodule has a significant likelihood for neoplasia $(11,12)$. Other classic features on CT that are more likely to be seen with malignant pulmonary nodules or masses include spiculated or lobulated margins as opposed to smooth margins, which are more likely to be seen in benign lesions (13).

With regard to prior studies that have evaluated combined CT and PET in the assessment of lung cancer, many studies have compared the diagnostic utility of one study as opposed to the other (14-16). There are no previous studies, which have evaluated the differences in uptake of FDG based on tumor type in conjunction with CT features. Therefore, the purpose of this study was two-fold: 1) to evaluate the relationship between variations in uptake of FDG measured by SUV and lung cancer cell subtype and 2) to determine if any features on CT combined with FDG-PET characteristics help to better identify lung cancers by tumor subtype.

\section{Materials and methods}

This study was approved by the human subject's research committee of our institution. A retrospective review of all FDG-PET datasets acquired for primary lung cancer diagnosis and staging over a 24-month period (May 2003 to May 2005) was performed. Patients were included who received a primary diagnostic or staging FDG-PET scan. Patients were excluded if they had received prior radiation or chemotherapy for their tumor, the tumor was massive or widely metastatic such that the primary focus could not be identified, or FDG-PET scan was performed more than one month prior to tissue diagnosis. 
Table I. Combined tumor features on CT and FDG-PET scans.

\begin{tabular}{lllll}
\hline Tumor features & BAC & Adeno non-BAC & Squamous cell & Large cell \\
\hline Number & 15 & 35 & 23 & 9 \\
Average size (cm) & $1.6 \pm 0.6$ & $2.7 \pm 1.5$ & $3.5 \pm 1.9$ & $3.2 \pm 1.4$ \\
Range (cm) & $0.7-2.6$ & $1-8.5$ & $2-5.8$ & $1.2-8.5$ \\
Averaged SUV & $1.6 \pm 1.2$ & $4.6 \pm 0.8$ & $9.2 \pm 1$ & $7.5 \pm 1.5$ \\
Range & $0.4-5.9$ & $0.4-11.6$ & $1.6-32.6$ & $2.9-19.1$ \\
Spiculated margin & $7 \%(1)$ & $51 \%(18)$ & $43 \%(11)$ & $78 \%(7)$ \\
Pure ground glass & $47 \%(8)$ & $9 \%(3)$ & $0 \%$ & $0 \%$ \\
Lobulated margin & $13 \%(2)$ & $29 \%(10)$ & $43 \%(10)$ & $22 \%(2)$ \\
Smooth margin & $0 \%$ & $3 \%(1)$ & $0 \%$ & $0 \%$ \\
Mix solid/ground glass & $33 \%(5)$ & $6 \%(2)$ & $0 \%$ & $0 \%$ \\
Atelectasis & $0 \%$ & $3 \%(1)$ & $13 \%(3)$ & $0 \%$ \\
Peripheral & $73 \%(11)$ & $63 \%(22)$ & $43 \%(10)$ & $44 \%(4)$ \\
Mid-lung & $20 \%(3)$ & $26 \%(9)$ & $26 \%(6)$ & $33 \%(3)$ \\
Central lung & $7 \%(1)$ & $11 \%(4)$ & $30 \%(7)$ & $22 \%(2)$ \\
\hline
\end{tabular}

Patient population. A total of 81 patients with 82 lung cancers were included in the study. Forty-seven patients were women and 34 were men. Their average age was $67 \pm 11$ years (range 35-90 years). The diagnosis of lung cancer was made either by percutaneous needle biopsy or surgical histopathology. Twenty-three patients had squamous cell carcinoma. Nine patients had large cell carcinoma. Fifty patients had adenocarcinoma. Fifteen of the patients in this group had the variant bronchioloalveolar cell carcinoma (BAC) (Table I). Five patients had adenocarcinoma with BAC features, which were included in the adenocarcinoma group. Pathology results that included tumor differentiation (poor, moderate or well) were available in 64 tumors.

FDG-PET and CT scans. All patients received a diagnostic and/or staging FDG-PET scan prior to surgical resection, biopsy or therapy. FDG-PET scans were obtained with an ECAT $\mathrm{HR}^{+}$(Siemens, Schaumburg, IL). Image spatial resolution was $4.5 \mathrm{~mm}$ full width half maximum (FWHM) with slice thickness of $2.0 \mathrm{~mm}\left(\mathrm{ECAT} \mathrm{HR}^{+}\right)$. The patients fasted for at least $6 \mathrm{~h}$ before scanning. FDG $(\sim 15 \mathrm{mCi})$ was administered intravenously as a bolus and static emission images were obtained 60 min later. All emission datasets were attenuation corrected. Transmission images acquired on the ECAT camera were measured with rotating rod sources loaded with Germanium-68. Image reconstruction was performed with ordered subset expectation maximization (OSEM) algorithms.

All patients received a separate thoracic CT scan on a GE LightSpeed scanner (GE, Milwaukee, WI) within 30 days of the PET study. Scans were obtained at $2.5-\mathrm{mm}$ slice. Sixtyseven patients received contrast enhanced CT scans with $100 \mathrm{ml}$ of Isovue 300 (Bracco Diagnostics, Princeton, NJ).

Study interpretation and analysis. PET and CT scans were jointly reviewed by two radiologists blinded to the pathologic

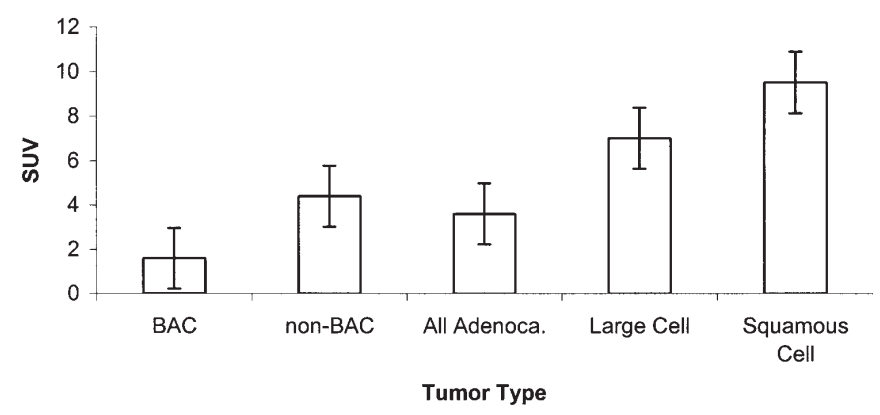

Figure 1. Standard uptake value (SUV), (mean \pm SEM) of lung cancers based on tumor type.

results. Interpretations were made by consensus. PET and CT datasets were reviewed and fused on a Reveal-MVS (Mirada Solutions Ltd., Oxford, UK) workstation. The dose, time of injection and body weight were used to calculate standardized uptake values (SUV). A small region-of-interest (ROI) was placed over each tumor on fused PET/CT datasets at the area of greatest activity. The SUV was calculated at the location of peak FDG uptake. Areas of necrosis or tumor regions with potential for partial volume averaging with adjacent normal lung parenchyma were avoided with guidance of fused CT images.

CT features analyzed included: location (central vs. midlung vs. peripheral), tumor size and margins/features. Each tumor was described with one of the following margins/ features: solid with spiculated, lobulated or smooth margins; pure ground glass (non-solid) (17) or mixed solid with ground glass (part-solid) (Table I). If a tumor had any component of ground glass and spiculated margins, it was categorized as partsolid rather than spiculated margins. A tumor was categorized as atelectatic if the margins were partly obscured by adjacent collapsed lung. 
A

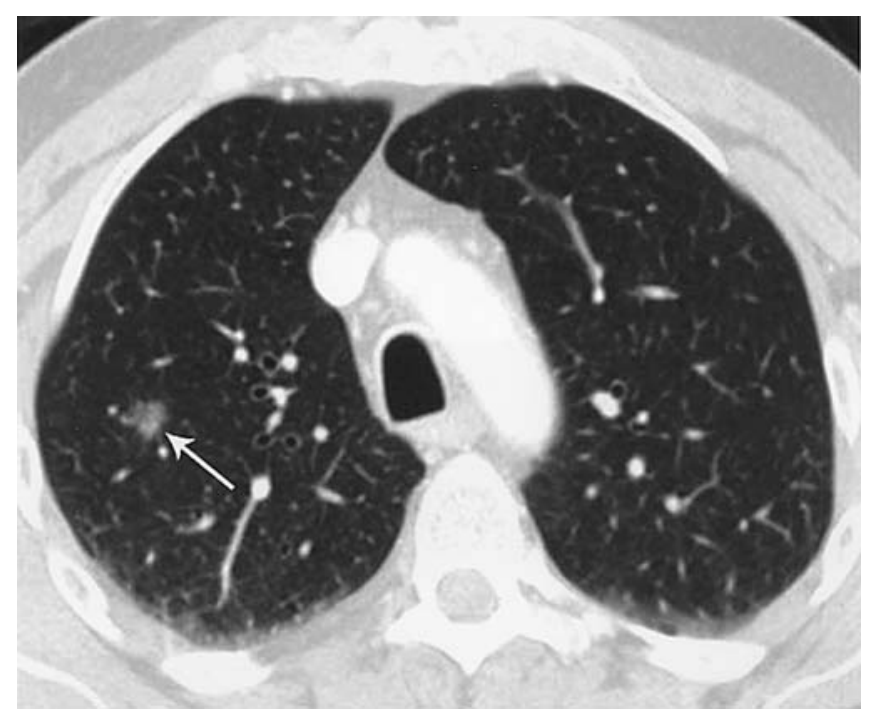

B

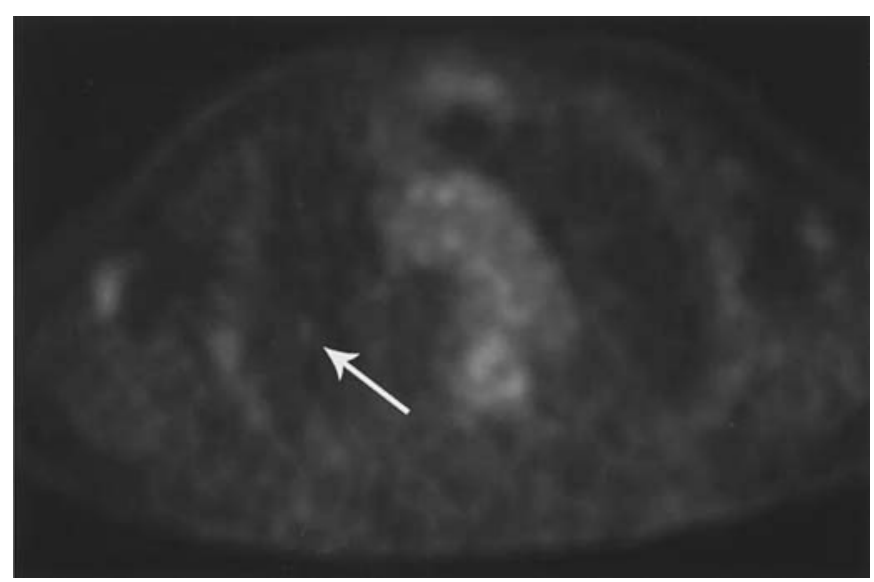

Figure 2. A 59-year-old man with BAC of the lung. (A) Axial CT image in lung windows shows non-solid ground glass nodule in right upper lobe (arrow). (B) Axial FDG-PET image shows peak uptake of SUV 0.4 (arrow).

Statistical analysis. One-way ANOVA was performed to compare mean SUV measurements between each tumor type. Fisher's exact test was used to determine the relationship between tumor type and the presence of tumor features or margins on CT. Logistic, using all terms and Stepwise logistic regression, was used to determine if combination of SUV and CT patterns better predicted tumor type.

\section{Results}

Peak SUV (mean \pm standard error) measurements based on tumor cell type were: all adenocarcinomas $3.7 \pm 1.5$ (range 0.4-11.6); adenocarcinoma BAC type 1.6 1.2 (0.4-5.9); adenocarcinoma non-BAC 4.6 \pm 0.8 (0.4-11.6); squamous cell 9.2 \pm 1 (1.6-32.6); large cell 7.5 \pm 1.5 (2.9-19.1) (Table I) (Fig. 1). Of the 35 non-BAC adenocarcinomas, 5 had mixed features with BAC. Their mean SUV was 1.5 $\pm 0.2(0.4-2.0)$.

One-way ANOVA showed significant differences between peak SUV and lung cancer tumor types $(\mathrm{p}<0.0001)$. Significant differences were found between all adenocarcinomas and squamous cell types $(\mathrm{p}<0.0001)$; all
A

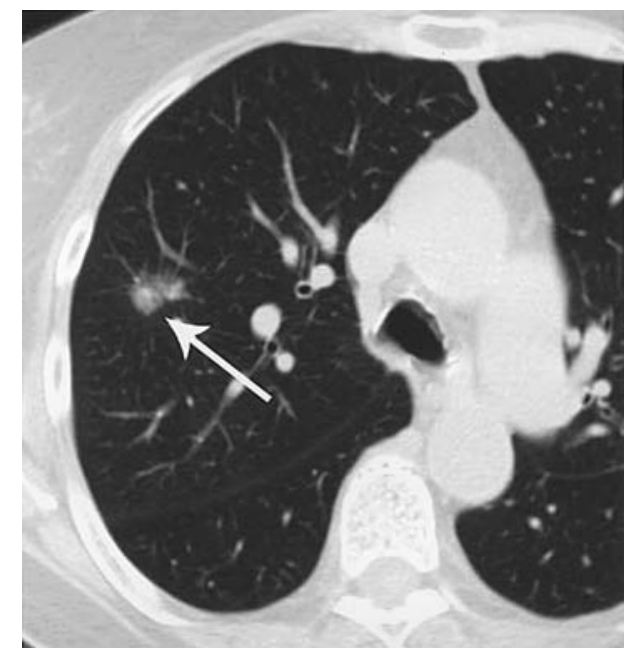

B

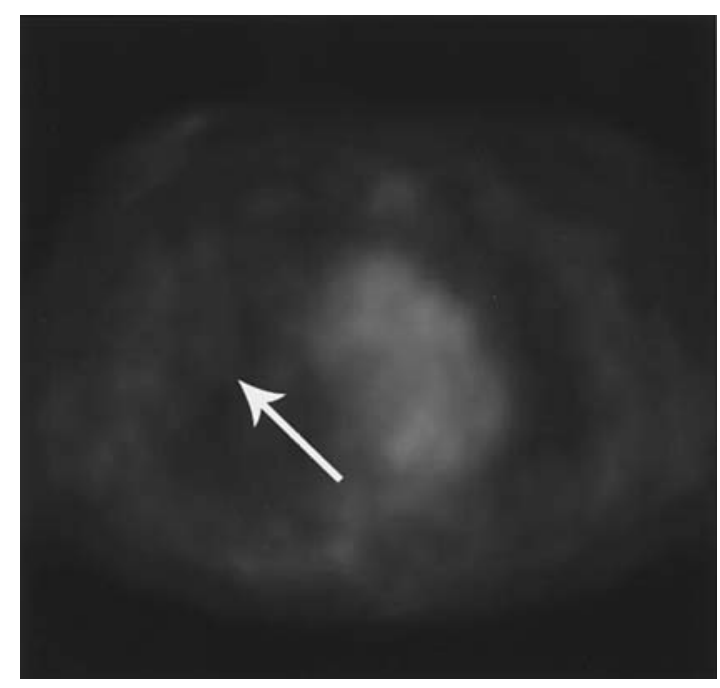

Figure 3. An 83-year-old woman with BAC of the lung. (A) Axial CT image in lung windows shows part-solid nodule in right upper lobe (arrow). (B) Axial FDG-PET image shows peak uptake of SUV 0.4 (arrow).

adenocarcinomas and large cell $(\mathrm{p}=0.03)$; non-BAC adenocarcinoma and squamous cell $(\mathrm{p}=0.0005) ; \mathrm{BAC}$ and non-BAC adenocarcinoma $(\mathrm{p}=0.04), \mathrm{BAC}$ and squamous cell $(\mathrm{p}<0.0001)$ and BAC and large cell $(\mathrm{p}=0.004)$. There was no significant difference in SUV between large cell carcinoma and squamous cell carcinoma or large cell carcinoma and non-BAC adenocarcinoma.

Fisher's exact test showed significant relationship between tumor type and the presence of ground glass in tumors. CT features of non-solid nodule (pure ground glass) $(\mathrm{p}<0.0003)$ (Fig. 2), and mixed solid/ground glass tumor $(\mathrm{p}<0.0001)$ (Fig. 3) were significantly related with BAC. All 3 non-BAC adenocarcinoma tumors that showed pure ground glass on $\mathrm{CT}$ had some BAC features. CT feature of spiculation was not a feature of BAC $(\mathrm{p}<0.04)$ but was seen in all other tumor types (Table I) (Fig. 4). Other nodule features, including associated atelectasis, smooth or lobulated margins were not distinguishing features for tumor subtypes.

Stepwise logistic regression analysis of SUV and CT values showed that SUV was a significant finding in distinguishing 
A

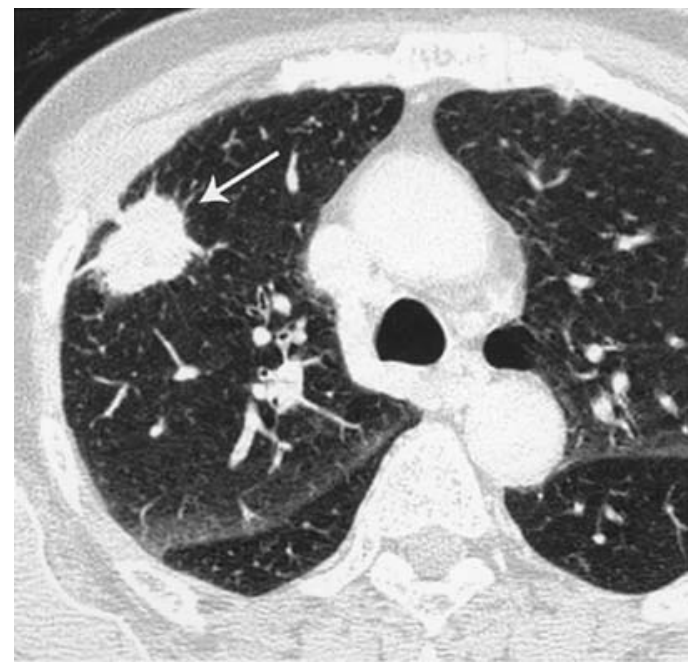

B

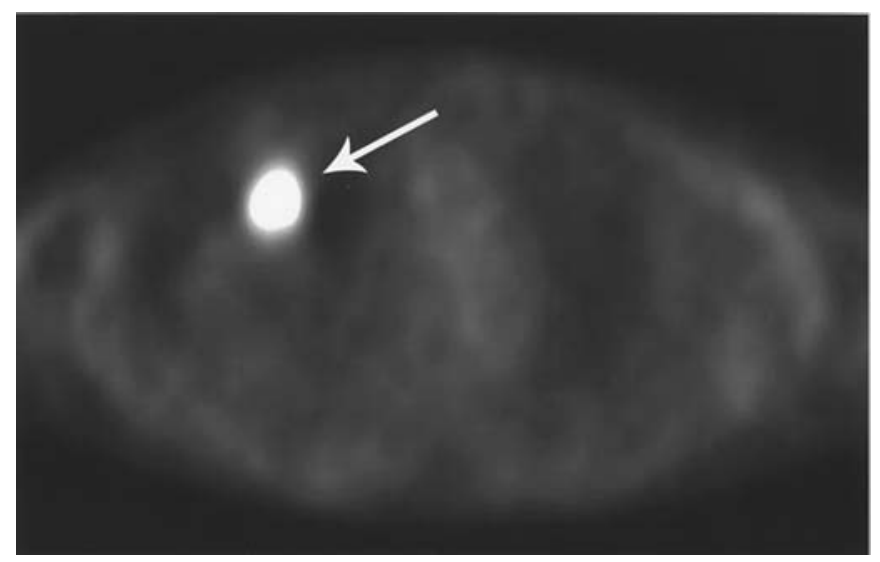

Figure 4. A 54-year-old man with squamous cell carcinoma of the lung. (A) Axial CT image in lung windows shows a spiculated nodule in right upper lobe (arrow). (B) Axial FDG-PET image shows peak FDG uptake of SUV 15.5 (arrow).

tumor types $(\mathrm{p}<0.008)$. Other features on CT did not further contribute to the distinction of tumor type once SUV results were utilized. There was no correlation between differentiation (poor, moderate, well), of tumor subtype and SUV.

\section{Discussion}

Cancer cells preferably utilize anaerobic glycolysis for energy (18) and rely on both increased glucose transport across the cell membrane (19) and enhanced hexokinase activity (20) to meet higher glucose demand.

Prior studies have reported on the variation of FDG uptake based on lung cancer grade 4 (21). Higashi et al found that FDG uptake varies with degree of differentiation of adenocarcinomas (6). They found that FDG uptake in BAC (SUV $1.36 \pm 0.82$ ) is significantly lower than that of well-differentiated adenocarcinoma (SUV 2.92 \pm 1.28 ) and moderately differentiated adenocarcinoma (SUV 4.63 \pm 1.86 ). In another study this group found that those adenocarcinomas with more invasive features (i.e. poorly differentiated tumors) had greater FDG uptake (SUV 4.36 \pm 1.94 ) compared to non-invasive tumors (SUV 1.53 \pm 0.88 ) (22). Additionally, they found that patients with tumors with greater FDG uptake had a poorer prognosis.

A significant difference in FDG uptake was found between the well-differentiated adenocarcinoma subtype BAC and other non-BAC adenocarcinomas including tumors in the latter group that were well differentiated. The adenocarcinomas with mixed features that included BAC had a peak SUV $(1.5 \pm 0.2)$ that was lower than all remaining non-BAC adenocarcinomas (SUV 3 \pm 1.5 ), even though one tumor was poor, 3 were moderate and one was well differentiated.

There was a significant difference in FDG uptake between all adenocarcinoma cell types (BAC and non-BAC) and squamous cell carcinoma or large cell carcinomas of any differentiation. Therefore, increased tumor FDG uptake does not always mean higher-grade or poorer prognosis $(23,24)$. A difference was also seen in the peak SUV value between large cell carcinoma and squamous cell although the value was not statistically significant.

Our results suggest that the uptake of FDG in tumors is multifactorial as also suggested by other studies. Various studies have shown that FDG uptake in tumor cells also varies with growth rate $(25)$, regional hypoxia $(19,26)$ and with Glut-1 concentration, one of the five cell membrane glucose transporters $(19,26,27)$. Brown et al (19) found that FDG uptake and Glut-1 expression were greater in squamous cell carcinoma than adenocarcinoma.

Because of the growing use of FDG-PET for lung nodule evaluation and the integration of CT and PET imaging with combined PET/CT scanning, we incorporated an analysis of CT patterns of focal lung cancers along with FDG uptake to determine if the combination of modalities improves the differentiation of tumor types. An ability to improve the radiologic distinction of tumor types could be helpful in situations in which a patient with suspected lung cancer is a poor candidate for invasive diagnostic procedures (i.e. severe emphysema, poor tolerance for complications of a pneumothorax due to severe cardiac disease) yet needs tumor cell distinction for therapy.

The only CT features that contributed significant information in distinguishing tumor subtypes was the presence of ground glass. Ground glass was significantly more common in BAC tumors $(\mathrm{p}<0.0003)$ and was present in 3 out of 4 patients with adenocarcinoma with BAC features. A solid tumor with spiculated margins was an infrequent feature of BAC but was present in all other subtypes including non-BAC adenocarcinomas $(\mathrm{p}=0.04)$.

Forty-seven percent of the BAC tumors in our group were non-solid (pure ground glass) and an additional $29 \%$ were mixed solid/ground glass. Namely, $76 \%$ of BAC tumors showed some ground glass feature. This is in contrast to $12 \%$ of non-BAC adenocarcinoma (again 3 of which had BAC features), and $0 \%$ of squamous cell and large cell tumors. Although the relationship between the presence of ground glass features on CT and BAC was extremely significant $(p<0.0003)$. This feature was not statistically contributory in the stepwise logistic regression. The difference in peak SUV values between BAC and other tumor types was far more significant in the stepwise logistic analysis and superseded any CT values as useful predictors of tumor type. 
In terms of practical applications, our results confirm that the peak FDG uptake of BAC tumors (average SUV 1.6 \pm 1.2 in our results) is clearly below the widely accepted SUV cut-off of 2.5 for malignancy (4). These results as well as those of other studies $(9,10,28)$ demonstrate a need to replace the concept 'negative' PET study with 'low FDG uptake' PET. With the growing use of combined PET/CT and readily available CT images, radiologists and referring clinicians should suspect BAC when CT demonstrates a persistent nodule with ground glass features despite low FDG uptake. These results emphasize that CT features of ground glass in a nodule (non-solid or part-solid) should raise a concern for BAC. Increased FDG should clearly raise the level of suspicion for malignancy, but low FDG uptake in a nodule with ground glass features should also be treated as potentially malignant. As previously described by Nakata et al, such nodules warrant further diagnostic evaluation by either biopsy or resection (12).

There are a few limitations in our study that are worth noting. Because of the retrospective nature of our study, information about tumor differentiation was not available for all patients and therefore only 64 tumors had these results. Our results do show that BAC, a well-differentiated subtype of adenocarcinoma had a significantly lower SUV than other tumor types; however, our sample size was too small to further distinguish subtle variations in SUV among squamous cell, large cell or non-BAC adenocarcinoma subtypes based on differentiation. A larger prospective analysis may potentially reveal more significant results. Furthermore, in tumors diagnosed by fine needle aspiration there is always the risk of sampling error; however, in the clinical setting these patients were managed based on the results of these biopsies.

In conclusion, SUV measurements for non-small cell lung cancer are significantly different between adenocarcinoma versus both squamous cell and large cell carcinoma. Further distinction can be made between the SUV of subtype BAC and all other NCLC cell types. These results suggest that lung cancer tumor cell subtypes express variation in glycolytic activity that is multifactorial and intensity of FDG uptake does not necessarily indicate poor differentiation as previously described. In addition, we conclude that low FDG uptake in nodules with ground glass features should not be interpreted as benign but require further diagnostic work-up for the presence of BAC.

\section{References}

1. Gupta NC, Maloof J and Gunel E: Probability of malignancy in solitary pulmonary nodules using fluorine-18-FDG and PET. J Nucl Med 37: 943-948, 1996.

2. Gupta NC, Frank AR, Dewan NA, et al: Solitary pulmonary nodules: detection of malignancy with PET with 2-[F-18]-fluoro2-deoxy-D-glucose. Radiology 184: 441-444, 1992.

3. Dewan NA, Shehan CJ, Reeb SD, Gobar LS, Scott WJ and Ryschon K: Likelihood of malignancy in a solitary pulmonary nodule: comparison of Bayesian analysis and results of FDGPET scan. Chest 112: 416-422, 1997.

4. Patz EF Jr, Lowe VJ, Hoffman JM, et al: Focal pulmonary abnormalities: evaluation with F-18 fluorodeoxyglucose PET scanning. Radiology 188: 487-490, 1993.

5. Herder GJ, Golding RP, Hoekstra OS, et al: The performance of (18)F-fluorodeoxyglucose positron emission tomography in small solitary pulmonary nodules. Eur J Nucl Med Mol Imaging 31: 1231-1236, 2004.

6. Higashi K, Ueda Y, Seki H, et al: Fluorine-18-FDG PET imaging is negative in bronchioloalveolar lung carcinoma. J Nucl Med 39: 1016-1020, 1998.
7. Heyneman LE and Patz EF: PET imaging in patients with bronchioloalveolar cell carcinoma. Lung Cancer 38: 261-266, 2002.

8. Erasmus JJ, McAdams HP, Patz EF Jr, Coleman RE, Ahuja V and Goodman PC: Evaluation of primary pulmonary carcinoid tumors using FDG PET. Am J Roentgenol 170: 1369-1373, 1998.

9. Kim BT, Kim Y, Lee KS, et al: Localized form of bronchioloalveolar carcinoma: FDG PET findings. Am J Roentgenol 170: 935-939, 1998.

10. Marom EM, Sarvis S, Herndon JE 2nd and Patz EF Jr: T1 lung cancers: sensitivity of diagnosis with fluorodeoxyglucose PET. Radiology 223: 453-459, 2002.

11. Libby DM, Smith JP, Altorki NK, Pasmantier MW, Yankelevitz D and Henschke CI: Managing the small pulmonary nodule discovered by CT. Chest 125: 1522-1529, 2004.

12. Nakata M, Saeki H, Takata I, et al: Focal ground-glass opacity detected by low-dose helical CT. Chest 121: 1464-1467, 2002.

13. Erasmus JJ, Connolly JE, McAdams HP, Roggli VL: Solitary pulmonary nodules: Part I. Morphologic evaluation for differentiation of benign and malignant lesions. Radiographics 20: 43-58, 2000

14. Gupta NC, Graeber GM, Rogers JS 2nd and Bishop HA: Comparative efficacy of positron emission tomography with FDG and computed tomographic scanning in preoperative staging of non-small cell lung cancer. Ann Surg 229: 286-291, 1999.

15. Pieterman RM, van Putten JW, Meuzelaar JJ, et al: Preoperative staging of non-small-cell lung cancer with positron-emission tomography. N Engl J Med 343: 254-261, 2000.

16. Berlangieri SU, Scott AM, Knight SR, et al: F-18 fluorodeoxyglucose positron emission tomography in the non-invasive staging of non-small cell lung cancer. Eur J Cardiothorac Surg 16 (Suppl 1): S25-S30, 1999.

17. Henschke CI, Yankelevitz DF, Mirtcheva R, McGuinness G, McCauley D and Miettinen OS: CT screening for lung cancer: frequency and significance of part-solid and nonsolid nodules. Am J Roentgenol 178: 1053-1057, 2002.

18. Golshani-Hebroni SG and Bessman SP: Hexokinase binding to mitochondria: a basis for proliferative energy metabolism. J Bioenerg Biomembr 29: 331-338, 1997.

19. Brown RS, Leung JY, Kison PV, Zasadny KR, Flint A and Wahl RL: Glucose transporters and FDG uptake in untreated primary human non-small cell lung cancer. J Nucl Med 40: 556-565, 1999.

20. Mathupala SP, Rempel A and Pedersen PL: Glucose catabolism in cancer cells. J Biol Chem 270: 16918-16925, 1995.

21. Vesselle H, Schmidt RA, Pugsley JM, et al: Lung cancer proliferation correlates with [F-18]fluorodeoxyglucose uptake by positron emission tomography. Clin Cancer Res 6: 3837-3844, 2000.

22. Higashi K, Ueda Y, Ayabe K, et al: FDG PET in the evaluation of the aggressiveness of pulmonary adenocarcinoma: correlation with histopathological features. Nucl Med Commun 21: 707-714, 2000.

23. Sugawara Y, Quint LE, Iannettoni MD, et al: Does the FDG uptake of primary non-small cell lung cancer predict prognosis? A Work in Progress. Clin Positron Imaging 2: 111-118, 1999.

24. Marom EM, Aloia TA, Moore MB, et al: Correlation of FDGPET imaging with Glut-1 and Glut-3 expression in early-stage non-small cell lung cancer. Lung Cancer 33: 99-107, 2001.

25. Duhaylongsod FG, Lowe VJ, Patz EF Jr, Vaughn AL, Coleman RE and Wolfe WG: Lung tumor growth correlates with glucose metabolism measured by fluoride-18 fluorodeoxyglucose positron emission tomography. Ann Thorac Surg 60: 1348-1352, 1995

26. Ito T, Noguchi $Y$, Satoh $S$, Hayashi H, Inayama $Y$ and Kitamura H: Expression of facilitative glucose transporter isoforms in lung carcinomas: its relation to histologic type, differentiation grade, and tumor stage. Mod Pathol 11: 437-443, 1998 .

27. Higashi K, Ueda Y, Sakurai A, et al: Correlation of Glut-1 glucose transporter expression with. Eur J Nucl Med 27: 1778-1785, 2000.

28. Yap CS, Schiepers C, Fishbein MC, Phelps ME and Czernin J: FDG-PET imaging in lung cancer: how sensitive is it for bronchioloalveolar carcinoma? Eur J Nucl Med Mol Imaging 29: 1166-1173, 2002. 